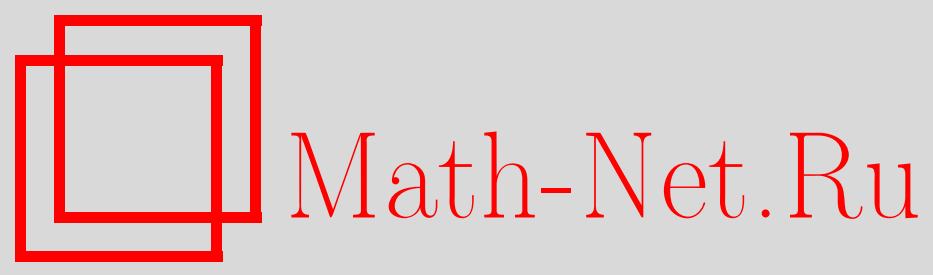

М.-М. Деза, М. И. Штогрин, Вложение химических графов в гиперкубы, Матем. заметки, 2000, том 68, выпуск 3, 339352

DOI: https://doi.org/10.4213/mzm951

Использование Общероссийского математического портала Math-Net.Ru подразумевает, что вы прочитали и согласны с пользовательским соглашением http://www . mathnet.ru/rus/agreement

Параметры загрузки:

IP : 54.166 .219 .16

26 апреля 2023 г., 15:28:58

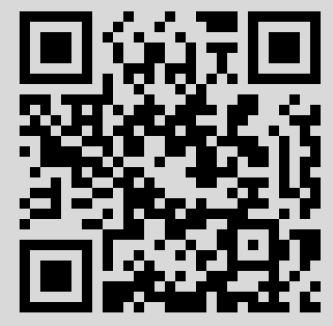




\title{
ВЛОЖЕНИЕ ХИМИЧЕСКИХ ГРАФОВ В ГИПЕРКУБЫ
}

\author{
М. Деза, М. И. Штогрин
}

\begin{abstract}
Рассматриваются реализованные на плоскости планарные графы, применяемые в химии: степени вершин 3 и 2 , все внутренние грани $r$-гоны, за исключением одной или двух других внутренних граней, а занимаемая внутренними гранями область плоскости односвязна. Для широких классов таких граффов решается вопрос о вложении графиической метрики их вершин в многомерные кубы или кубические решетки с сохранением или же удвоением всех расстояний. Попутно дается полная классификация некоторых интересных семейств таких графов.
\end{abstract}

Библиография: 22 названия.

Мы рассматриваем здесь односвязные полигональные системы. Это плоские графы, состоящие из контуров многоугольников (впредь называемых внутренними гранями), заполняющих связную односвязную область, причем любые два многоугольника либо вовсе не пересекаются, либо пересекаются по ребру. Значит, степень каждой вершины графа, расположенной внутри области, равна 3 , а каждой вершины на границе области -2 или 3. Эти графы рассматриваются в органической, физической и математической химии, где они представляют полициклические сопряженные углеводороды или полициклические ароматические углеводороды. Потребовав односвязности области, мы запретили в ней дыры (исключая таким образом, например, короноиды), т.е. рассматриваем (в химических терминах) полностью конденсированные полициклические ароматические углеводороды $C_{n} H_{m}$, где $n$ - число всех вершин (атомов углерода $C$ ), a $m$ - число вершин степени 2 (атомов водорода $H$ ). В зависимости от того, имеет ли граф вершины, являюшиеся внутренними точками области, или нет, в химических терминах он называется пери- или ката-конденсированным. Случай конечного 3-связного химического графа $C_{n} H_{0}$ по теореме Штейница всегда соответствует реберному остову многогранника. Но мы в основном концентрируемся на случае, когда имеются вершины степени 2 , т.е. когда число атомов водорода не равно нулю.

Назовем химический граф, взятый вместе со всеми внутренними гранями, полициклом, а точнее поли-r-uиклом, если контуры всех внутренних граней являются комбинаторными $r$-угольниками. В случае полицикла, как установлено в [1], по заданному графу однозначно определяются его $r$-циклы как все минимальные циклы графа. Назовем граф моно-q-r-uиклом, если контуры всех внутренних граней, за исключением одного $q$-угольника, являются $r$-угольниками. Наконец, $\partial u-q-r$-иикл означает, что два

Работа выполнена при финансовой поддержке Российского фонда фундаментальных исследований, грант № 99-01-00010, и Фонда поддержки ведущих научных школ, грант № 00-15-960111. 
$q$-угольника и $r$-угольники являются всеми внутренними гранями. Эти три класса покрьвают большую часть химических графов, рассматриваемых в химической литературе (см., например, [2]-[5] и многочисленные ссылки оттуда, из которых мы взяли химическую терминологию).

Примеры химических графов - прототипов соответствующих серий - даны на рис. 1 .
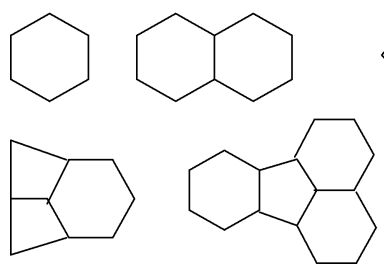
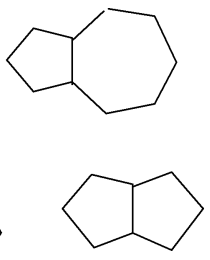
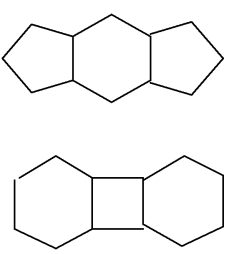

Рис. 1. Примеры химических графов: бензол $C_{6} H_{6}$, нафрталин $C_{10} H_{8}$, азулен $C_{10} H_{8}$, индацен $C_{12} H_{8}$, терфенилоид $C_{9} H_{5}$, флуорантен $C_{16} H_{10}$, пентален $C_{8} H_{6}$, бифенилин $\mathrm{C}_{12} \mathrm{H}_{8}$

Задача, которую мы здесь рассматриваем, состоит в том, чтобы в данном классе химических графов найти все влоэс имы е в гиперкуб $\mathbb{H}_{d}$ или полукуб $\frac{1}{2} \mathbb{H}_{d}, d \leqslant \infty$. А именно, мы ищем, можно ли адресовать вершины графа двоичными последовательностями длины $d$ так, чтобы расстояние Хэмминга между двумя вершинами равнялось расстоянию между этими вершинами в графе или же этому удвоенному расстоянию (т.е. всегда с одной и той же шкалой -1 или 2 , см. [6]). Полиномиальный алгоритм, проверяющий возможность такого вложения, дан в [7]. Методы нахождения таких вложений для планарных графов указаны в [6] и [8].

В математических терминах вложимость графов в гиперкубы (с какой-то шкалой на все расстояния) равносильно изометрическому вложению графа в пространство $l_{1}^{k}$. Такие вложения рассматривались в математической химии только для бензеноидов [9]; в теореме 3 мы дадим широкое обобщение этого результата, включающее все поли-6-циклы. В [9] указьвается также почему такие вложения представляют интерес для химиков: для вычисления параметров, основанных на расстояниях (число Винера, т.е. сумма всех попарных расстояний в графе, и т.д.), и в целях номенклатуры.

Односвязный поли-6-цикл имеет еще два названия: фузен и полигекс. Он также назьвается соответственно бензеноидом или гелииеном в зависимости от того, является ли он подграфом гексагонального разбиения $\left(6^{3}\right)$ плоскости или нет. По аналогии назовем $r$-геличеном для $r=3,4,5, r \geqslant 7$ тот поли- $r$-цикл, которьй не является частичным подграфом реберного остова соответственно тетраэдра, куба, додекаэдра или разбиения плоскости Лобачевского на $r$-угольники со схождениями по 3 в каждой вершине, т.е. разбиения $\left(r^{3}\right)$. Всякий $r$-гелицен допускает непрерывное локально гомеоморфное клеточное отображение в $\left(r^{3}\right)$ (см. [1]).

Назовем поли- $r$-цикл собственным, если он не $r$-гелищен, т.е. является частичным подграфом регулярного разбиения $\left(r^{3}\right)$ сферы при $r=3,4,5$, евклидовой плоскости при $r=6$, плоскости Лобачевского при $r \geqslant 7$. Все несобственные поли- $r$-циклы являются $r$-гелищенами. Собственные поли- $r$-циклы могут быть изометрическими подграфами разбиения $\left(r^{3}\right)$ или нет. В первом случае они вложимы. В последнем случае они подразбиваются на вложимые или нет. Например, есть ровно $1+1+3+7+23$ поли-6-циклов 
с $p_{6}=1,2,3,4,5$. Все они собственные; первый гелицен появляется при $p_{6}=6$. Среди этих 35 поли-6-циклов ровно 8 не изометричны подграфам в $\left(6^{3}\right)$ : см. рис. $2 \mathrm{a}$, шесть его расширений по ребрам $c, f, g, h$ или по паре ребер $(a, b),(d, e)$, и рис. $2 \mathrm{~b}$. Заметим, что все конечные вложимые поли- $r$-циклы вложимы в $\mathbb{H}_{d / 2}$, если $r$ четно, или в $\frac{1}{2} \mathbb{H}_{d}$, если $r$ нечетно (где при отсутствии замкнутых зон, состоящих из внутренних ребер, $d-$ периметр заполняемой ими области, т.е. длина границы внешней грани).

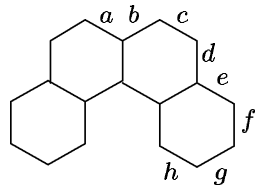

a<smiles>C1CCC2C(C1)CCC1CC3CCC4CCCCC4C3CC12</smiles>

$\mathrm{b}$

Р ис. 2. Все неизометрические подграфы $\left(6^{3}\right)$ с $p_{6} \leqslant 5$

Перед началом доказательств введем необходимые технические обозначения и понятия. $G \rightarrow \mathbb{H}_{d}, G \rightarrow \frac{1}{2} \mathbb{H}_{d}, G \rightarrow \mathbb{Z}_{d}, G \rightarrow \frac{1}{2} \mathbb{Z}_{d}, d \leqslant \infty$, будет означать, что граф $G$ со шкалой $\lambda=1$ или $\lambda=2$ вкладывается в гиперкуб $\mathbb{H}_{d}$ или (если он бесконечен) в кубическую решетку $\mathbb{Z}_{d} ;$ коэффициент $\frac{1}{2}$ означает, что при вложении графа все расстояния в нем удваиваются. Далее, $G-e, G-v$ означает граф, полученный выбрасьванием из $G$ одного ребра или вершины; $P_{n}, P_{\mathbb{N}}, P_{\mathbb{Z}}$ означает реберный путь с вершинами $\{1,2, \ldots, n\}$, или $\mathbb{N}$ (натуральные числа), или $\mathbb{Z}$ (все целые числа) соответственно; $G \times G^{\prime}$ означает прямое (декартово) произведение графов $G$ и $G^{\prime}$; через $\alpha_{3}, \beta_{3}, \gamma_{3}$, Dо и Iсо мы обозначаем соответственно тетраэдр, октаэдр, куб, додекаэдр и икосаэдр (и, говоря о графах, их одномерные остовы).

Важным необходимым условием вложимости графа является его 5-гональность [10]: для любых пяти его вершин $x, y, a, b, c$ справедливо следующее 5-гональное неравенство:

$$
\rho_{x y}+\left(\rho_{a b}+\rho_{a c}+\rho_{b c}\right) \leqslant\left(\rho_{x a}+\rho_{x b}+\rho_{x c}\right)+\left(\rho_{y a}+\rho_{y b}+\rho_{y c}\right) .
$$

Все примеры невложимости в нашей работе получены указанием в графе 5 точек, нарушаюших это неравенство. На рисунках вершины $a, b, c$ отмечены кружочками, а $x, y-$ квадратиками.

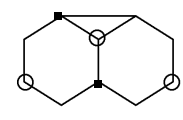

a

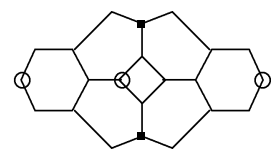

$\mathrm{b}$

Рис. 3. Запрещенные изометрические подграфы вложимых моно-3- и моно-4-фузенов

Графы, которые мы здесь рассматриваем, в некотором смыслепротивоположны классу тех планарных графов, у которых степени всех внутренних вершин больше 3 и которые не содержат треугольников; в [11] доказана вложимость таких графов. Заметим, что граф, состоящий из сходящихся в одной тройной вершине 3 -угольника, $m$-угольника и 


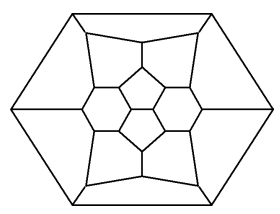

Рис. 4. Фуллерен $F_{26} \rightarrow \frac{1}{2} \mathbb{H}_{12}$. Центральньй подграф $\Gamma_{1,1}$ не 5-гонален и неизометричен

$n$-угольника, вложим тогда и только тогда, когда $m$ и $n$ одновременно нечетны [12]; нарушение 5 -гонального неравенства $(*)$ при $m=n=6$ иллюстрирует рис. 3а.

Примерами односвязных полигональных систем являются униформные разбиения евклидовой плоскости $\left(6^{3}\right) \rightarrow \mathbb{Z}_{3},\left(4.8^{2}\right) \rightarrow \mathbb{Z}_{4},(4.6 .12) \rightarrow \mathbb{Z}_{6}$, не 5-гональное $\left(3.12^{2}\right)$, а также униформные разбиения гиперболической плоскости $\left(r^{3}\right) \rightarrow \mathbb{Z}_{\infty}$ при четном $r$ и $\left(r^{3}\right) \rightarrow \frac{1}{2} \mathbb{Z}_{\infty}$ при нечетном $r$. Конечно, под определение подходят также взятые без одной грани многогранники $\alpha_{3} \rightarrow \frac{1}{2} \mathbb{H}_{4}, \gamma_{3} \rightarrow \mathbb{H}_{3}$, Do $\rightarrow \frac{1}{2} \mathbb{H}_{10}$, усеченный октаэдр вложи́м в $\mathbb{H}_{6}$, а также не 5-гональные усеченные многогранники $\alpha_{3}, \gamma_{3}$, Dо и Iсо. В качестве примера см. на рис. 4 вложимьй в $\frac{1}{2} \mathbb{H}_{12}$ граф фуллерена $F_{26}$ (многогранника с вершинами степени 3, с 12 пятиугольными и 3 шестиуголньми гранями). Этот пример интересен также следующими особенностями: наличием неальтернированных зон (см. определение перед теоремой 2) и тем, что не 5-гональный подграф $\Gamma_{1,1}=C_{14} H_{8}$, введенньй в замечании 4 (ii) и данный на рис. $5 \mathrm{~b}$, является здесь неизометрическим подграфом вложимого фуллерена на рис. 4.

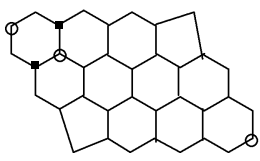

a

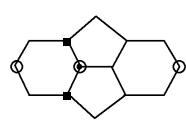

b

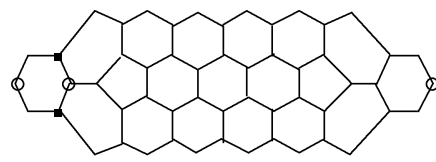

C

Рис. 5. Запрещенные изометрические подграфы вложимых ди-5-фузенов

Основным параметром рассматриваемых плоских графов, как и в случае многоугольников, будет их $p$-вектор $p=\left(p_{3}, p_{4}, \ldots\right)$, где $p_{i}$ означает число комбинаторньх $i$-циклов, т.е. $i$-угольников среди всех внутренних граней.

В настоящей статье рассматриваются следующие девять случаев (напомним, что $p_{i}$ обозначает число внутренних $i$-граней):

1) поли-3-циклы: $p_{3}$ любое, остальные $p_{i}=0$;

2) поли-4-циклы: $p_{4}$ любое, остальные $p_{i}=0$;

3) поли-5-циклы: они введены в [2];

4) $p_{3}=p_{4}=0, p_{5} \leqslant 1$, остальные $p_{i}$ любые;

5) моно-3-6-циклы: $p=\left(p_{3}=1, p_{6}\right)$;

6) бифениленоиды: $p=\left(p_{4}=1, p_{6}\right)$;

7) ди-3-6-циклы: $p=\left(p_{3}=2, p_{6}\right)$;

8) терфениленоиды: $p=\left(p_{4}=2, p_{6}\right)$;

9) индансеноиды: $p=\left(p_{5}=2, p_{6}\right)$. 
В теореме 1 случаи 1) и 2) будут полностью исследованы. В теореме 2 дан список всех запрещенных изометрических подграфов вложимого поли-5-цикла, т.е. случай 3). В теореме 3 доказывается вложимость для широкого класса химических графов, данных в случае 4). Этот случай включает, например, поли-6-циклы и азуленоиды $p=\left(p_{5}=1, p_{7}\right)$. Вложимость бензеноидов, т.е. фузенов, являющихся изометричными подграфами в $\left(6^{3}\right)$, в гиперкубы была доказана в [9]. Теорема 4 характеризует все вложимые моно- $q$-фузены $(q=3,4,5)$, т.е. случаи 5$)$ и 6$)$, а также ди-3-фузены, т.е. случай 7$)$. В теореме 5 даны некоторые необходимые условия вложимости ди- $q$-фузенов с $q=4,5$, точнее, в случаях 8) и 9 ) приводится список запрещенных изометрических подграфов (их бесконечно много). Высказывается предположение, что условия теоремы 5 не только необходимы, но и достаточны для вложимости терфениленоидов и индансеноидов. Наконец, в теореме 6 указаны все моноэдральные полипентагоны.

Все планарные графы без внутренних вершин (т.е. ката-конденсированные) вложимы (см. [6, предложение 3]). Более того, характеризация вложимых поли-5-циклов и ди-5-фузенов запрещенньми подграфами показывает, что любой такой граф вложи́м, если имеет ровно одну внутренюю вершину. Каждьй поли-5-цикл, в котором все внутренние вершины изолированы друг от друга (т.е. любые две вершины не могут быть соединены реберным путем, состоящим только из внутренних точек), вложи́м; и вложи́м в $\frac{1}{2} \mathbb{Z}_{\infty}$, если число изолированных внутренних вершин бесконечно (см. бесконечный граф на рис. 6а). С другой стороны, существуют не вложимые ди-3-фузены (т.е. ди-3-6-циклы) с единственной внутренней вершиной (см. рис. 6b).

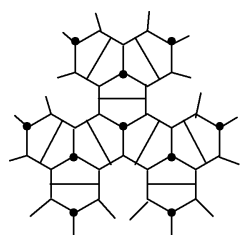

a

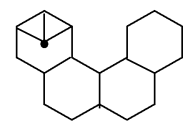

$\mathrm{b}$

Рис. 6. Примеры пери-конденсированных графов:

a) $\rightarrow \mathbb{Z}_{\infty} ;$ b) не 5-гонален

Для доказательства вложимости графов мы ниже используем достаточные условия вложимости планарного графа, данные в [6, предложение 2]. А именно, пусть граф таков, что множество его ребер разложимо на зоны (последовательности противоположных ребер граней, альтернирующие направления в случае нечетных граней), так что каждая зона соответствует одному из измерений куба, в которьй вкладывается граф. Зоны дают маркировку ребер (одним или двумя измерениями в зависимости от того, является ли граф двудольньм или нет). Затем, приняв одну из вершин за начало, можно маркировать каждую другую вершину симметрической разностью маркированнных ребер на каком-либо кратчайшем пути от выбранного начала. В силу предполагаемой односвязности области, заполняемой внутренними гранями графа, такая маркировка не зависит от выбора пути, связьвающего маркируемую точку с заданным началом. При этом для изометричности такого отображения требуется выпуклость разреза, соответствующего любой альтернированной зоне (см. [6]).

ТЕорема 1. (i) Все поли-3-иикль: $\alpha_{3},\left(\alpha_{3}-e\right) \rightarrow \frac{1}{2} \mathbb{H}_{4}$ (неизометричный подграф тетраэдра $\left.\alpha_{3}\right),\left(\alpha_{3}-v\right)=\alpha_{2}$. 
(ii) Все поли-4-иикль: $\gamma_{3},\left(\gamma_{3}-e\right)$ (не 5-гонален), $\left(\gamma_{3}-v\right) \rightarrow \mathbb{H}_{3},\left(P_{2} \times P_{n}\right) \rightarrow \mathbb{H}_{n}$, $\left(P_{2} \times P_{\mathbb{N}}\right) \rightarrow \mathbb{Z}_{2},\left(P_{2} \times P_{\mathbb{Z}}\right) \rightarrow \mathbb{Z}_{2}$. Подграфы куба: $\gamma_{3},\left(\gamma_{3}-v\right),\left(P_{2} \times P_{n}\right)$ nри $n=2,3,4 ;$ изометрические среди них: $\gamma_{3},\left(\gamma_{3}-v\right),\left(P_{2} \times P_{2}\right)=\gamma_{2},\left(P_{2} \times P_{3}\right)$.

Доказательство осуществляется простой проверкой.

ЗАмЕЧАнИЕ 1 . Есть $1+1+2+4+7+18$ поли-5-циклов с $p_{5}=1,2,3,4,5,6$. Среди этих 33 поли-5-гонов можно выделить следующие.

(i) 5-гелиценом (т.е. полипентагоном, который не является подграфом додекаэдра) является один с $p_{5}=5$ и семь с $p_{5}=6$; а именно, граф на рис. $7 \mathrm{a}$, четыре его расширения по ребрам $e, f$ или паре ребер $(a, b),(c, d)$ и 3 остальные на рис. $7 \mathrm{~b}, 7 \mathrm{c}, 7 \mathrm{~d}$ (все они вложимы).

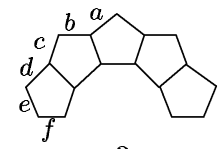

a

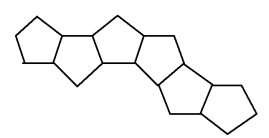

$\mathrm{b}$

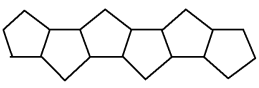

c

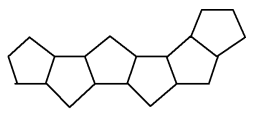

d

Рис. 7. 5-гелицены с $p_{5} \leqslant 6$

(ii) Ровно 5 графов из этих 33 являются изометрическими подграфами додекаэдpa: $G_{1}$ - реберньй остов одного 5 -угольника (т.е. цикл $C_{5}$ ), $G_{2}$ - реберньй остов двух 5 -угольников, смежных по ребру, $G_{3}$ - реберньй остов трех 5-угольников додекаэдра, сходящихся в одной вершине, $G_{4}$ - реберньй остов четырех 5-угольников додекаэдра, инцидентных с одним его ребром, $G_{5}$ - реберньй остов шести 5-угольников додекаэдра, пять из которых инцидентны с шестым. Иными словами, подграф додекаэдра изометричен тогда и только тогда, когда он не содержит граничного ребра, инцидентного трем 5-угольникам. Более того, эти 5 графов исчерпывают все нетривиальные изометрические подграфы додекаэдра; конечно, все они вложимы.

(iii) Ровно 2 из этих 33 графов не вложимы (не 5-гональные конфигурации указаны на рис. 8).
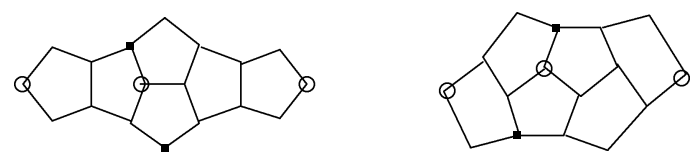

Рис. 8. Запрещенные подграфы вложимых поли-5-циклов

(iv) Все поли-5-циклы, являющиеся подграфами додекаэдра, даны в [2, рис. 3-5]. Среди четырнадшати оставшихся подграфов додекаэдра, т.е. случаев $7 \leqslant p_{5}, 5$-гональны (более того, вложимы) ровно четыре из них [2, рис. 5]: три неизометричных подграфа додекаэдра $C_{20} H_{8} \rightarrow \frac{1}{2} \mathbb{H}_{17}, C_{20} H_{8} \rightarrow \frac{1}{2} \mathbb{H}_{17}, C_{19} H_{7} \rightarrow \frac{1}{2} \mathbb{H}_{15}$, см. рис. 9 , и реберный остов самого Do.

(v) Реберный остов Dо имеет два особых (неизометрических и невложимых) подграфа Do $-v \subset$ Do $-e \subset$ Do, расширяемых только согласно указанным здесь включениям, и сам он не является подграфом никакого другого полицикла.

ЗАмЕчАниЕ 2. (i) Пример поли-5-цикла, вложимого только в $\frac{1}{2} \mathbb{Z}_{\infty}-$ бесконечная сильносвязная цепочка пятиугольников, которая служит универсальной накрьвающей 

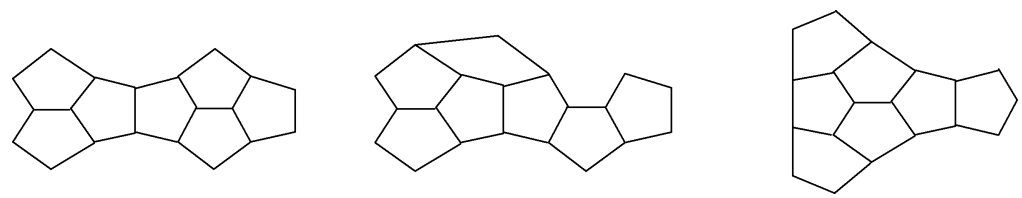

Рис. 9. Все вложимые неизометрические подграфы додекаэдра с $p_{5} \geqslant 7$

конечной замкнутой цепочки, состоящей из пяти пятиугольников, окружающих фиксированный пятиугольник; этот граф, как и граф, изображенньй на рис. 6а, содержит дерево с бесконечным числом ветвей.

(ii) Любой отличньй от додекаэдра односвязный поли-5-цикл с не более чем одной точкой степени 2 является бесконечным.

(iii) Если поли- $r$-цикл при $r \geqslant 6$ имеет не более $r-1$ вершин степени 2 , то он бесконечен. При $r=3,4,5$ конечные поли- $r$-циклы $\alpha_{3}-e, \gamma_{3}-e$, Do $-e$ имеют по две вершины степени 2 , а $\alpha_{3}-v, \gamma_{3}-v$, Do $-v$ имеют по три вершины степени 2 . При $r \geqslant 5$ существуют бесконечные поли- $r$-циклы, количество вершин степени 2 которых равно любому наперед заданному натуральному числу.

Ниже нам понадобится еще два определения.

Полипентагон, т.е. поли-5-цикл, соответствующий графу $G$, мы обозначаем через $\boldsymbol{\Pi}(G)$. (По сути, $\Pi(G)$ есть клеточное разбиение диска, обладающее реберным остовом $G$. Как оказалось (см. [1], [13] и [14]), абстрактное устройство полипентагона П $(G)$ однозначно определяется абстрактным устройством графа $G$. Поэтому довольно часто мы называем полициклом сам граф $G$.) Соединим любой пентагон из $\Pi(G)$ с любым другим его пентагоном некоторой цепочкой пентагонов, в которой оба они являются первым и последним, причем любые два следующие друг за другом пентагона цепочки смежны по общей стороне. Назовем $k$-окрестностью данного пентагона совокупность всех тех пентагонов из $\Pi(G)$, для каждого из которых кратчайшая цепочка, соединяюшая его с данньм пентагоном, содержит не более $k+1$ пентагонов. Тогда в 0-окрестность пентагона входит только он сам, в 1-окрестность пентагона входит он сам и его соседи по сторонам, в 2-окрестность входит он сам, его соседи по сторонам и соседи соседей. В частности, если какой-то пентагон имеет в $\Pi(G)$ ровно пять соседей, то его 1-окрестность представляет собою $\Pi\left(G_{5}\right)$ (см. замечание $\left.1(\mathrm{ii})\right)$.

У любого ребра $n$-угольника на плоскости есть одно противоположное ребро, если $n$ четно, и два (левое и правое) противоположные ребра, если $n$ нечетно. Назовем зоной разбиения плоскости последовательность ребер (возможно, замкнутую) такую, что каждое последующее ребро является противоположным предыдущему. Назовем зону альтернирующей, если в соотвествующей последовательности выборов (между левым и правым ребром) выбор меняется каждый раз.

Tеорема 2. Не совпадающий с Dо поли-5-иикл $G$ вложи́м в $\frac{1}{2} \mathbb{H}_{d}$ (әде $d-n е р и-$ метр) тогда и только тогда, когда он не содерэит подграфов, приведенных на рис. 8, не всегда изометрических.

Невложимость графов из рис. 8 дана указанием на каждом из них 5 вершин, нарушающих 5-гональное неравенство. Такое нарушение имеет место для всякого изометрического, но не для всякого неизометрического подграфа. В последнем случае нарушение 
5-гонального неравенства может оказаться при другой расстановке 5 вершин, а не той, которая указана на рис. 8 .

ДОКАЗАТЕЛЬСТВО ТЕОРЕМЫ 2. Граф $G_{5}$, введенный в замечании 1(ii), удовлетворяет условиям теоремы 2 , он вложи́м в $\frac{1}{2} \mathbb{H}_{10}$.

Пусть, далее, $G \neq G_{5}$, но у полипентагона $\Pi(G)$ есть пентагон, у которого 1-окрестность имеет вид $\Pi\left(G_{5}\right)$. Тогда 2-окрестность этого пентагона может быть только одной из следующих семи (в каждой из них содержится подграф из рис. 8): П(Do $\left.-v_{1}-v_{2}-v_{3}\right)$, $\boldsymbol{\Pi}\left(\mathrm{Do}-v_{1}-v_{2}\right), \boldsymbol{\Pi}(\mathrm{Do}-v-e), \boldsymbol{\Pi}\left(\mathrm{Do}-e_{1}-e_{2}\right), \boldsymbol{\Pi}(\mathrm{Do}-v), \boldsymbol{\Pi}(\mathrm{Do}-e), \boldsymbol{\Pi}(\mathrm{Do})$. Три последние 2-окрестности встречаются только у трех индивидуальных полипентагонов $\Pi(\mathrm{Do}-v), \boldsymbol{\Pi}(\mathrm{Do}-e), \boldsymbol{\Pi}(\mathrm{Do})$, из которых только П(Do) вложи́м. В любом другом полипентагоне $\Pi(G)$ у тех пентагонов, чья 1-окрестность имеет вид $\Pi\left(G_{5}\right), 2$-окрестность может быть только одного из оставшихся четырех видов. Но каждый из соответствующих четырех графов не 5-гонален и поэтому невложим. Докажем изометричность каждого из них. В 2-окрестности имеется не более трех соседей второго порядка. Число соседей третьего порядка может быть равно только 1 или 2. Расширив 2-окрестность до 3-окрестности, в каждом конкретном случае легко убедиться, что реберньй граф 2-окрестности является изометрическим подграфом реберного графа 3-окрестности, что следует из отсутствия у $\mathbf{\Pi}(G)$ некоторых соседей второго порядка окружаемого пентагона и из односвязности $\Pi(G)$. Более того, по этим же причинам реберньй граф 2 -окрестности остается изометрическим подграфом во всем $G$. Следовательно, любой графф $G \neq G_{5}$ с условиями $G_{5} \subset G \neq$ Dо невложим.

Покажем, что при условиях теоремы 2 альтернирующая зона не может быть замкнутой. В самом деле, предположим обратное, пусть полипентагон П $(G)$ имеет замкнутую альтернирующую реберную зону. Тогда она охватывает в $\Pi(G)$ подграф $G_{3}-$ три сходящиеся в одной вершине пентагона (см. замечание 1(ii)). Каждьй из них имеет 1-окрестность вида $\Pi\left(G_{5}\right)$. Такой полипентагон всегда совпадает с одним их трех полипентагонов $\mathrm{Do}-v, \mathrm{Do}-e, \mathrm{Do}$, содержащих подграфы из рис. 8 . А для них условия теоремы 2 не выполнены.

Покажем теперь, что при условиях тероемы 2 любой разрез, альтернирующий все ребра зоны в $\Pi(G)$, является выпуклым.

Пусть $k$ - число пентагонов альтернирующей зоны. Если $k \geqslant 5$, то примыкающий к зоне пентагон (если такой имеется) обязан быть смежным с концевым (левым или правым - ведь зона не замкнута) ее пентагоном, так как в противном случае имелся бы запретньй граф из рис. 8а (из отсутствия запретного графа из рис. 8а следует, что вдали от концов зоны ее край одновременно является краем самого полицикла П $(G)$; сказанное относится к обоим краям зоны). Пентагон, смежньй с концевьг, может иметь одно из трех не запрещенных положений, в которых он может быть смежньм с тремя, двумя или только одним пентагоном зоны (в последнем случае он смежен не по ребру зоны, иначе ее длина стала бы $k+1)$.

Если $k \geqslant 6$, то все три способа соседства пентагона с данной зоной при каждом ее конще допустимы, причем даже все одновременно: см. рис. 10а при $k=6$ и рис. 10b при $k=7$.

Если $k$ четно, то оба края зоны имеют одну и ту же длину. При наличии смежного по трем сторонам зоны пентагона (при четном $k$ для любого края зоны имеется лишь один такой пентагон), заменив эти три стороны на две другие стороны пентагона, укоротим ломаную между концами края, с которьм инцидентен пентагон, на 1 по сравнению с 


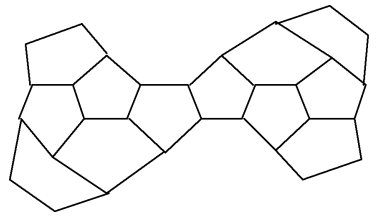

a

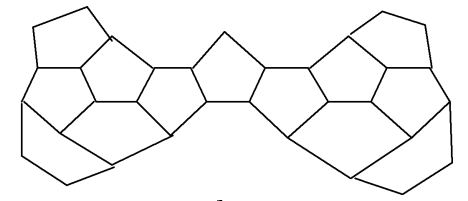

$\mathrm{b}$

Рис. 10. Зоны длины $k=6$ и $k=7$ со всевозможньми соседними пентагонами

длиной края. Так как вблизи середины зоны оба конща ее края принадлежат краю $\Pi(G)$, а полипентагон $\Pi(G)$ односвязен, то более короткой ломаной между концами края зоны быть не может. Следовательно, при наличии пентагона, смежного по трем сторонам зоны, кратчайшее расстояние между концами зоны на 1 меньше длины края зоны. В силу сказанного при четном $k$ возможно одно из трех:

a) оба края зоны геодезические;

b) один край геодезический, а другой нет, причем кратчайшая между концами другого края на 1 ребро короче его длины и лежит в своей области разреза;

c) оба края не геодезические; кратчайшее расстояние между концами одного края равно кратчайшему расстоянию между концами другого края (так как оно на 1 короче длины самого края, а длины краев равны), и каждая кратчайшая лежит в своей области разреза.

Во всех трех случаях разрез рассматриваемого графа по ребрам зоны является выпуклым.

Если $k$ нечетно, то один край зоны всегда геодезический и он на 1 короче другого края, который в свою очередь может быть:

a) геодезическим;

b) не геодезическим, а кратчайшая между его концами на 1 короче края и лежит в своей области разреза;

c) не геодезическим, а кратчайшая между его концами на 2 ребра (так как имеется два пентагона, смежные с зоной по трем сторонам из этого края) короче длины края, причем лежит в своей области разреза.

И в этом случае разрез является выпукльм. В самом деле, даже в случае с) если прибавить два ребра к кратчайшей между концами края, чтобы мысленно перевести коншы через зону в другую область разреза, то получим путь, длина которого равна длине этого края. Но длина второго (геодезического) края на 1 короче длины этого пути. Следовательно, перевести концы в другую область разреза и получить между новыми концами более короткое расстояние невозможно.

Аналогично поступаем в случаях $1 \leqslant k \leqslant 5$. Так, например, при $k=5$ из двух вакансий на смежные по трем сторонам с зоной ввиду запрета рис. $8 \mathrm{~b}$ допустим только один пентагон. Поэтому следует рассмотреть два случая: есть один пентагон, смежньй с зоной по трем сторонам, или ни одного. Все остальные вакансии допустимы, причем даже одновременно. В каждом случае разрез по ребрам зоны является вьпуклым. При $k=4$ из двух вакансий на смежных по трем сторонам пентагонов допусти́м только один из них, а при наличии такового из двух других смежных с ним вакансий опять допустима лишь одна. Все остальные вакансии допустимы. В каждом случае разрез является выпукльм. Так же и в оставшихся случаях $k=3,2,1$. 
Итак, на каждое ребро пентагона приходится по две зоны. Каждый разрез вьпуклый. Каждой зоне поставим в соответствие свою координатную ось. В итоге получим отображение полипентагона в гиперкуб со шкалой $\lambda=2$. Теорема 2 доказана.

ТЕорема 3. Односвязный химический граф без треугольников, без четырехугольников и не более чем с одним пятиугольником вложиім. В частности, при $r \geqslant 6$ все поли-г-ииклы вложимы.

Доказательство теоремы 3 основано на методах работы [6], а именно, все разрезы графа, соответствующие альтернирующим зонам, как легко это проверить при условиях теоремы 3, являются выпуклыми.

Теорема 4. (i) Моно-3-фузены (один треугольник, остальные шестиугольники) вложимь (в $\frac{1}{2} \mathbb{H}_{d}$, где $d$ - периметр) тогда и только тогда, когда одна из вериин треугольника имеет степень 2. Другими словами, эти графы вложимы тогда и только тогда, когда они не содержат изометрического подграфа, данного на puc. $3 \mathrm{a}$.

(ii) Ди-3-фузен (два треугольника, остальные 6-угольники) вкладывается тогда и только тогда, когда каждый треугольник имеет вершину степени 2.

(iii) Моно-5-фузены (флуореноиды) всегда вложимы (в силу теоремы 3).

(iv) Моно-4-фузень (бифениленоиды) вложимы тогда и только тогда, когда они не содержат изометрического подграфа, данного на рис. $3 \mathrm{~b}$.

ДОКАЗАТЕЛЬСТВО ТЕОРЕМЫ 4. Если в случаях (i), (ii) имеется треугольник, степень каждой вершины которого равна 3 , то граф не может быть вложен ввиду наличия запретного подграфа, данного на рис. За. Если же треугольник имеет вершину степени 2 , то после удаления каждого из таких треугольников мы окажемся в условиях теоремы 3 и получим вложимьй подграф. Восстановление треугольника с вершиной степени 2 (одного или двух) никак не может нарушить вложимость графа.

Подчеркнем, что два шестиугольника, не смежные с 4-угольником (рис. 3b), инцидентны двум противоположнымм висячим ребрам из вершин 4-угольника.

Если в случае (iv) какая-то зона была бы не вьпуклой, то в такую зону входили бы только шестиугольники, причем кратчайшее расстояние между двумя вершинами какого-то ее края проходило бы по ломаной, которая заходит в другую область разреза, которая не примыкает к данному краю. Замкнем ломаную, прибавив к ней ребра края. Полученная замкнутая ломаная окружает 4-угольник. Не нарушая обшности, можно считать, что рассматриваемая зона касается 4-угольника. Однако исходная кратчайшая не может касаться 4-угольника, так как в противном случае кратчайшей была бы ломаная, идущая по самому краю зоны. Следовательно, 4-угольник с одной стороны примькает к краю зоны, а с другой стороны его окружают еще два 6-угольника, а в таком случае мы имеем запретньй подграф, изображенный на рис. $3 \mathrm{~b}$. Теорема 4 доказана.

Теорема 5. (i) Для влохимости ди-5-фузена (индансеноида) необходимо, чтобы он не содержсл зависящих от двух параметров $s, t \in \mathbb{N}$ запрещенных изометрических подграфов $\Gamma_{s, t}$ (см. рис. 5a, соответствующий параметрам $s=3 u t=2$, и рис. 5b, соответствующий параметрам $s=t=1)$ и зависящего от одного параметра $u \in \mathbb{N}$ запрещенного изометрического подграфа $\Gamma_{u}$ (см. рис. $5 \mathrm{c}$, соответствуюший параметру $и=3$ ). 
(ii) Для вложимости ди-4-фузена (терфениленоида) необходимо, чтобы он не содержал зависящих от двух параметров $s, t \in \mathbb{N}$ запрещенных изометрических подграфов $\Gamma_{s, t}^{\prime}$ (см. рис. 11a, соответствующий параметрам $s=3 u t=2, u$ рис. $11 \mathrm{~b}$, соответствующий параметрам $s=t=1$ ), зависящий от одного параметра $u \in \mathbb{N}$ запрещенный изометрический подграф $\Gamma_{u}^{\prime}$ (см. рис. 11с, соответствующий параметру $u=4$, и рис. $11 \mathrm{~d}$, соответствующий параметру $u=1) u$ запрещенный подграф, изображсенный на рис. $3 \mathrm{~b}$.

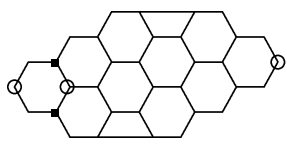

a

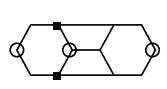

$\mathrm{b}$

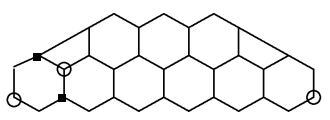

C

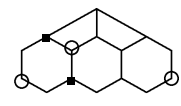

d

Рис. 11. Запрещенные изометрические подграфы вложимых ди-4-фузенов

Необходимость условий теоремы 5 очевидна, так как все изометрические подграфы, запрещенные в формулировке теоремы, не 5-гональны (на рисунках указаны пять точек, нарушающих условие 5-гональности).

Мы полагаем, что для вложимости ди-4-фузена (терфениленоида) и ди-5-фузена (индансеноида) условия теоремы 5 не только необходимы, но и достаточны.

ЗАмЕчАниЕ 3. Было бы интересно охарактеризовать плоские фуллерены, т.е. разбиения плоскости на комбинаторные пентагоны и гексагоны, так что каждая вершина имела бы степень 3. Ясно, что в таком разбиении число гексагонов всегда бесконечно и что для числа пентагонов $p_{5}=0$ и $p_{5}=1$ такой плоский фуллерен единственен, а для $p_{5}=2$ их бесконечно много (см. рис. 5). Нас интересует также, какой диск, состоящий из гексагонов и не более чем 6 пентагонов, дополняется гексагонами до разбиения всей плоскости (со всеми вершинами степени 3). Полипентагоны $\boldsymbol{\Pi}(\mathrm{Do}), \boldsymbol{\Pi}(\mathrm{Do}-e), \boldsymbol{\Pi}(\mathrm{Do}-v)$, например, а также гелищены не могут быть дополнены гексагонами до разбиения всей плоскости (со всеми вершинами степени 3 ). Из теоремы А. Д. Александрова [15] следует, что плоский фуллерен с $p_{5}$ пентагонами существует тогда и только тогда, когда $p_{5} \leqslant 6$. Аналогично, разбиение плоскости на 3 -угольники (4-угольники) и гексагоны с вершинами степени 3 существует тогда и только тогда, когда $p_{3} \leqslant 2$ (соответственно $\left.p_{4} \leqslant 3\right)$. Это следует из того, что любому такому разбиению плоскости можно поставить в соответствие изоморфньй двумерный полиэдр, составленный из правильных евклидовых многоугольников. Кривизна полиэдра с данной метрикой не отрицательна (она строго положительна лишь в некоторых вершинах полиэдра). Такой полиэдр по теореме Александрова допускает вложение в трехмерное пространство в виде вьпуклой поверхности. Полная кривизна вьпуклой поверхности, гомеоморфной плоскости, не превышает $2 \pi$. Доля $k$-угольника в кривизне равна $(6-k) \pi / 3$. Для поверхности из 6 - и $k$-угольников имеем $p_{k}(6-k) \pi / 3 \leqslant 2 \pi$, т.е. $p_{k} \leqslant 6 /(6-k)$.

ЗАмечание 4. (i) Среди множества молекулярных графов, данных в справочнике [16], только следующие не вложимы (более того, не 5-гональны): № 43, рис. 3.1, №o 43, 44, 46, 48-50, 105-112, 132-136, 168-176, 194-201 (примеры, содержащие треугольник с вершинами степени 3, см. его рис. 3.2). Даже небольшие запрешенные индансеноиды, данные нами на рис. 5 , не встретились в справочнике [16]. Как пример вложений 
для остальных молекул из [16] заметим, что коранулен $C_{20} H_{10} \rightarrow \frac{1}{2} \mathbb{H}_{15}$, декасилен $\mathrm{C}_{36} \mathrm{H}_{18} \rightarrow \frac{1}{2} \mathbb{H}_{33}$; т.е. только кекулен $\mathrm{C}_{48} \mathrm{H}_{24}$ не 5-гонален.

(ii) Одним из возможных направлений является характеризация вложимых графов $C_{m} H_{n}$ в данном классе, например, индансеноидов с $p=\left(p_{5}=2, p_{6}\right)$. Примеры синтезированных индасеноидов: ииркофульвален (называемый также полу-бакминстерфуллереном) $C_{30} H_{12}$, см. рис. $12 \mathrm{a}, C_{30} H_{14}, C_{26} H_{12}$. В [16] даны все 45 индансеноидов $C_{30} H_{12}$. Например, все четыре индансеноида $C_{15} H_{9}$, данных в [4, табл. 1], вложимы в $\frac{1}{2} \mathbb{H}_{15}$. Но данньй на рис. $5 \mathrm{~b}$ граф $C_{14} H_{8}$ не 5-гонален. А среди четырех индансеноидов $C_{30} H_{12}$ с симметрией $D_{2} h$, указанных в той же таблице, три, включая полу-бакминстерфуллерен, не 5-гональны (см. рис. $12 \mathrm{a}, 12 \mathrm{~b}, 12 \mathrm{c})$. Четвертьй, представленный на рис. $12 \mathrm{~d}$, вложи́м в $\frac{1}{2} \mathbb{H}_{20}$. Кстати, дуальньй граф циркофульвалена (т.е. дуальньй графф внутренних граней) вкладьвается в $\frac{1}{2} \mathbb{H}_{8}$.

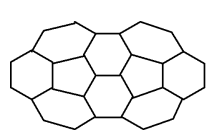

a

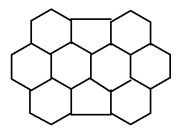

b

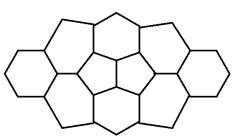

C

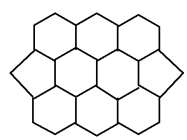

d

Р ис. 12. Четыре индансеноида $C_{30} H_{12}$ с симметрией $D_{2 h}$

Графы, изображенные на рис. $12 \mathrm{a}$ и рис. $12 \mathrm{~b}$, содержат запрешенный изометрический подграф $\Gamma_{2,1}$ из рис. 5а. Граф, изображенньй на рис. $12 \mathrm{c}$, содержит запрещенньй изометрический подграф $\Gamma_{1,1}$ из рис. $5 \mathrm{~b}$.

(iii) Многогранники с вершинами степени 3 , с $p=\left(p_{5}, p_{6}, p_{7}\right)$ и $12+p_{7}=p_{5}$ назьваются фуллероидами. Оба минимальных икосаэдральных фуллероида на 260 вершинах (см. [17]) не 5-гональны.

ЗАмЕчАниЕ 5. Вложенньй в плоскость граф $G$ однозначно задает в ней все внутренние грани, которые вместе составляют некоторую замкнутую область $\Omega(G)$. Если область $\Omega(G)$ односвязна, все внутренние грани суть $r$-угольники, причем любые две из них либо не пересекаются, либо имеют одно общее ребро, то разбиение области $\Omega(G)$ внутренними гранями, которое мы обозначили через $\Pi(G)$, представляет собою поли- $r$-цикл. Это значит, что в каждой вершине графа $G$, являющейся внутренней точкой области $\Omega(G)$, сходится ровно три $r$-угольника.

Сейчас мы рассмотрим примеры графов другого типа.

(i) Степень вершины равна 4. Рассмотрим следующее моно- $q$-4-гональное разбиение плоскости. Каждому ребру правильного $q$-угольника приставим бесконечную цепочку квадратов и каждый из возникших углов заполним ромбами. Граф этого разбиения вложи́м в $\mathbb{Z}_{q / 2}$ при четном $q$ и в $\frac{1}{2} \mathbb{Z}_{q}$ при нечетном $q$.

(ii) Степень вершины равна 4, 3,5. На рис. 13 дается пример двух вложимых бесконечных поли-4-циклов. Один (см. рис. 13a) не является проекцией никакого изометрического подграфа из $\mathbb{Z}_{d}, d<\infty$, но это зоноэдральное разбиение, комбинаторно эквивалентное $\mathbb{Z}_{2}$. Другой (см. рис. $13 \mathrm{~b}$ ) вкладьвается в $\mathbb{Z}_{d}$ только при $d=\infty$.

ЗАМЕЧАНИЕ 6. Назовем гексагональным тором (или торическим бензеноидом) нормальное разбиение поверхности тора на 6-угольники; степень каждой вершины равна 3. Такие торы рассматриваются, например, в [18]. Обозначим через $T_{i}$ такие разбиения тора на $i$ шестиугольников; ясно, что они имеют по $2 i$ вершин. $T_{i}$ многогранно (т.е. 


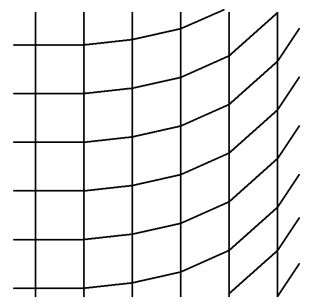

a

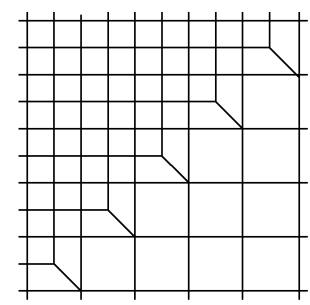

b

Рис. 13. Примеры необычных бесконечных поли-4-циклов: а) $\rightarrow \mathbb{Z}_{2} ;$ b) $\rightarrow \mathbb{Z}_{\infty}$

все грани суть вьпуклые полигоны, составляющие замкнутое связное 2-многообразие) только при $i \geqslant 7$. Единственная 7-вершинная триангулящия тора (Császár torus) имеет остов $K_{7}$ и является в $\mathbb{R}^{3}$ минимальным многогранником рода 1 [19].

Примеры: бензол-тор $T_{3}=K_{3,3}$, граф Хивуда $T_{7}$, пирен-тор $T_{8}$, коронен-тор $T_{12}$. ( $T_{7}$ реализует минимальную раскраску тора на 7 красок.) Все они не 5 -гональны, но куб $\gamma_{3}$ может быть реализован как $T_{4}$. Кстати, все неприводимые (в смысле [20]) квадрангулящии тора (разбиения тора на 4-угольники) имеют реберные графы $K_{5}$, $K_{6}-3 K_{2}, K_{4,4}, K_{3,6}, K_{4,5}-P_{3}, K_{5,5}-5 K_{2}, K_{7}-C_{7}$ (см. [20]). Первые два графа вложимы (как остовы $\alpha_{4}$ и $\beta_{3}$ ), а остальные не 5-гональны. Среди 10 неприводимых квадрангулящий бутылки Клейна (см. [21]) две вложимы (точнее, $Q^{6}$ и $Q^{7}$ имеют соответственно такие же остовы, как вложимые $\beta_{3}$ и $\alpha_{3}$ с пирамидами над двумя гранями), а остовы восьми остальных не 5-гональны. (Неприводимые квадрангулящии сфферы суть $C_{4}$, а проективной плоскости $-K_{4}, K_{3,4}$; из них только последний невложим.)

ЗАмЕчаниЕ 7. Графы в замечании 6 (а также кекулен из замечания 4(i)) являются примерами неодносвязны $x$ поли-6-циклов. Шесть неодносвязных поли-6-циклов даны $[22$, рис. 2] в качестве возможных гидрокарбонов; все они не 5-гональны.

Теорема 6. Существует ровно 8 моноэдральных полипентагонов. Из них три конечных: $G_{1}, G_{2}, G_{3}$ (см. замечание $1(\mathrm{ii})$ ), и пять бесконечных: бесконечная в обе стороны альтернирующая зона, бесконечная в обе стороны нидде не альтернирующая зона, бесконечная в обе стороны зона со строгим чередованием альтернирования и неальтернирования, склеенные вместе две неальтернирующие зонь, $а$ также полипентагон из рис. $6 \mathrm{a}$.

Доказательство теоремы 6 получается перебором всех 1-окрестностей и соответствующим дополнением их до моноэдральных полипентагонов. Лишь в этих случаях 1-окрестности всех пентагонов в полипентагоне одинаковы. Напомним, что поли- $r$-цикл назьвается моноәдральным, если его групша симметрии транзитивна на $r$-циклах.

В заключение приведем пример серии с растущей шкалой вложимых графов, предел которых невложим. Возьмем $i$ точек $A_{j}$ с натуральной координатой $j$ на оси, $1 \leqslant j \leqslant i$. Две соседние точки $A_{j-1}$ и $A_{j}$ примем за пару антиподальных вершин гипероктаэдра $\beta_{n_{j}}$ размерности $n_{j}=2^{j}$, дополнительно привлекая еще $2^{j-1}$ новых пар антиподов (чтобы пересекались только гипероктаэдры с антиподами в смежных парах $\left(A_{j-1}, A_{j}\right)$ и $\left(A_{j}, A_{j+1}\right)$, имея лишь одну общую вершину $\left.A_{j}\right)$. Все эти гипероктаэдры вместе составляют гирлянду $W_{i}$. Гипероктаэдр $\beta_{n_{i}}$, достроенньй на последней паре $\left(A_{i-1}, A_{i}\right)$, 
со шкалой $\lambda_{i}=2^{i-1}$ вложи́м в $\mathbb{Z}_{m_{i}}$, где $m_{i}=2^{i}$. В ту же решетку и с той же шкалой вложима вся гирлянда $W_{i}$. Предел $W_{i}$ при $i \rightarrow \infty$ обозначим через $W_{\infty}$. Однако $\lambda_{i} \rightarrow \infty$ при $i \rightarrow \infty$; следовательно, вложение предельной гирлянды $W_{\infty}$ с конечной шкалой невозможно даже в $\mathbb{Z}_{\infty}$.

\section{СПИСОК ЦИТИРОВАННОЙ ЛИТЕРАТУРЫ}

[1] Deza M., Shtogrin M. Polycycles // Voronoi Conference on Analytic Number Theory and Space Tilings (Kiev, September 7-14, 1998). Abstracts. Kiev, 1998. P. 19-23.

[2] Cyvin S. J., Cyvin B. N., Brunvoll J., Brendsdal E., Zhang Fuji, Guo Xiofeng, Tosic R. Theory of polypentagons // J. Chem. Inf. Comput. Sci. 1993. V. 33. P. 466-474.

[3] Cyvin S. J., Cyvin B. N., Brunvoll J. General formulations for some polycyclic hydrocarbons: di- $q$-polyhexes // Chemical Physics Letters. 1995. V. 240. P. 601-604.

[4] Dias J.R. Indacenoid isomers of semibuckmisterfullerene (Buckybowl) and their topological characteristics // J. Chem. Inf. Comput. Sci. 1995. V. 35. P. 148-151.

[5] Brunvoll J., Cyvin S. J., Cyvin B. N. Azulenoids // MATCH. 1996. V. 34. P. 91-108.

[6] Chepoi V., Deza M., Grishukhin V. Clin d'oeil on $L_{1}$-embeddable planar graphs // Discrete Appl. Math. 1997. V. 80. P. 3-19.

[7] Deza M., Shpectorov S. Recognition of $l_{1}$-graphs with complexity $O(\mathrm{~nm})$, or football in a hypercube // Europ. J. Combinatorics. 1996. V. 17. P. 279-289.

[8] Деза М., Штогрин М. И. Изометрические вложения полуправильных многогранников, разбиений и им дуальных в гиперкубы и кубические решетки // УМН. 1996. Т. 51. №6. C. $199-200$.

[9] Klavzar S., Gutman I., Mohar B. Labelling of bensenoid systems which reflects the vertex-distance relations // J. Chem. Inf. Comput. Sci. 1995. V. 35. P. 590-593.

[10] Тылкин М. Е. О геометрии Хэмминга единичных кубов // Докл. АН СССР. 1960. Т. 134. № 5. C. 1037-1040.

[11] Присакарь К.Ф., Солтан П.С., Чепой В. Д. О вложимости планарных графов в гиперкубы // Изв. АН МССР. Сер. матем. 1990. № 1. С. 43-50.

[12] Deza M., Tuma J. A note on $l_{1}$-rigid planar graphs // Europ. J. Combinatorics. 1996. V. 17. № 2, 3. P. 157-160.

[13] Деза М., Штогрин М. И. Примитивные полициклы и гелицены // УМН. 1999. Т. 54. № 6. С. $159-160$.

[14] Штогрин М. И. Примитивные полициклы: критерий // УМН. 1999. Т. 54. №6. С. 177-178.

[15] Александров А. Д. Выпуклые многогранники. М.-Л.: Гостехиздат, 1950.

[16] Dias J. R. Handbook of Polycyclic Hydrocarbons. Part B: Polycyclic Isomers and Heteroatom Analogs of Bensenoid Hydrocarbons: Elsevier, 1988.

[17] Dress A., Brinkmann G. Phantasmagorical fulleroids // MATCH. 1996. V. 33. P. 87-100.

[18] Hosoya H., Okuma Y., Tsukano Y., Nakada K. Multilayered cyclic fence graphs: novel cubic graphs related to the graphite network // J. Chem. Inf. Comput. Sci. 1995. V. 35. P. 351-356.

[19] Császár A. A polyhedron without diagonals // Acta Sci. Math. 1949. V. 13. P. 140-142.

[20] Nakamoto A. Irreducible quadrangulations of the torus // J. Combin. Theory. Ser. B. 1996. V. 67. P. 183-201.

[21] Nakamoto A. Irreducible quadrangulations of the Klein bottle // Yokohama Math. J. 1995. V. 43. P. $125-139$.

[22] Balaban A. T. Chemical graphs: Looking back and glimpsing ahead // J. Chem. Inf. Comput. Sci. 1995. V. 35. № 3. P. 345.

Ecole Normale Supérieure et CNRS (Paris)

Поступило

Математический институт им. В. А. Стеклова РАН

07.05.1999

Исправленный вариант

31.01 .2000 